\title{
Unitless Physics II: Internal Proton Structure US9-2
}

\author{
James W. Christy \\ 7285 Golden Eagle Drive, Flagstaff, AZ, USA \\ Email: jwc915@aol.com
}

How to cite this paper: Christy, J.W. (2017) Unitless Physics II: Internal Proton Structure US9-2. Journal of Modern Physics, 8, 1633-1649.

https://doi.org/10.4236/jmp.2017.89097

Received: June 16, 2017

Accepted: August 28, 2017

Published: August 31, 2017

Copyright $\odot 2017$ by author and Scientific Research Publishing Inc. This work is licensed under the Creative Commons Attribution International License (CC BY 4.0).

http://creativecommons.org/licenses/by/4.0/

\section{(c) (i) Open Access}

\begin{abstract}
The internal structure of the proton is chaotic according to the Standard Model. This paper explores several possibilities, based on US9-1, for producing an internal structure of the proton which is orderly. The hypothesis that quantized distance determination via particle to particle communication is required for force application eliminates $\mathrm{E} \& \mathrm{M}$ in the proton interior enabling a structure consisting of gravitational orbits. Communication velocities much greater than the velocity of light are required to enable the fundamental particles to generate the accepted laws of physics. In order to generate increasing complexity in Nature, the Uncertainty Principle must become the Organizational Principle. The Hydrogen atom is the source of evolution.
\end{abstract}

\section{Keywords}

Unitless Physics, Fundamental Particle Communication

\section{Introduction}

This paper presents ideas concerning the internal structure of the proton. These ideas are enabled by the unitless system developed in US9-1. Familiarity with US9-1 is required in the following presentation [1]. The primary constants of US9-1, $\underline{m}_{e}, R$ and @, adequately describe the actual values of mass, lifetime, and mass times lifetime of fundamental particles. This unexpected correlation should lead directly to internal algorithms. In $\underline{\mathrm{US}} 9, \underline{m}_{e}$ and @ are components of internal temporal processing in particles. US9 has entered the heart of the Standard Model.

Therefore, a primary objective of US9-2 is to produce a preliminary internal model of the proton which will provide practitioners of the Standard Theory a stable proton to interact with. 
The evolution of Physics in the Universe must emphasize efficiency of information processing and communication. The Hydrogen atom has the inherent capability of evolving to more complex structures, the atoms and molecules. This leads to biological evolution and thus must exhibit similar traits. The proton must contain fundamental properties that are shared by all evolved creatures. The experience of life is shared by all entities in the Universe. The Uncertainty Principle will become the Organizational Principle [2].

This results in internal time which can appear as external space-time but is simply a consequence of internal information processing. Force fields are communications between particles which originate within the particles but require a distance determination to be activated.

In US9-1, a unitless Physics was derived from fundamental constants which demonstrated intimate relationships in many areas of Physics that were not recognizable with the standard system. US9-1 produced a simple relationship between electrostatics and gravity and simple relationships between the constants of US9-1 and the masses and lifetimes of fundamental particles. Standard Physics concludes that the internal structure of the proton is chaotic [3].

US9-2 will consider the internal structure of the proton as the stable source of communications and physical law. We will introduce several principles which are required to answer logical gaps in the Standard Model and to provide connections in Physics to evolution. Such connections require an internal viewpoint of particles in addition to field theory. The purpose of this paper is to illuminate the properties necessary for a complete theory of Physics which provides internal mechanisms for the creation of communication fields and the reaction of fundamental particles to fields.

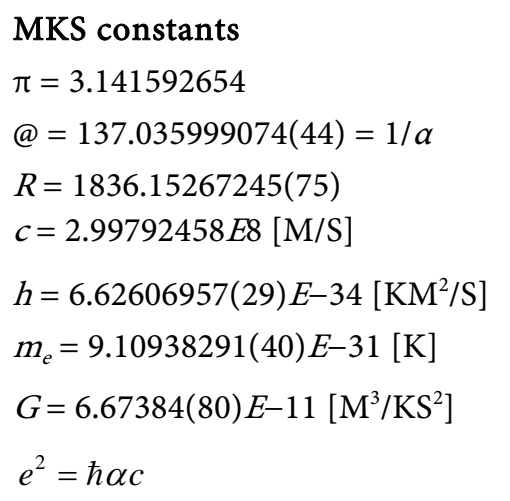

$$
\begin{aligned}
& \text { US9 constants } \\
& \pi=3.141592654 \\
& \text { @=137.035999074(44) } \\
& R=1836.15267245(75) \\
& \underline{c}=\underline{m}_{e} @ \\
& \underline{\hbar}=\underline{m}_{e}^{3} \\
& \underline{m}_{e}=3.6249118 E 6 \text { electron mass } \\
& \underline{G}=1 / R \underline{m}_{e}^{4}=1 / \underline{M}_{p} \underline{\hbar}=1 / R \underline{e}^{2} \\
& \underline{e}=\underline{m}_{e}^{2}
\end{aligned}
$$

US9 values are always underlined.

All physical constants are intimately related in US9, based on the value of the electron mass. A value of " 1 " has the same meaning for length, time and mass. The US9 values are assumed to be for internal processing where units are temporal.

US9 conversion constants:

$$
\begin{gathered}
K_{M}=1.4598347 E-17[\mathrm{M}] \\
K_{K g}=2.5129944 E-37[\mathrm{Kg}] \text { or }\left(0.141 \mathrm{ev} / c^{2}\right)
\end{gathered}
$$




$$
\begin{gathered}
K_{S}=2.41888433 E-17[\mathrm{~S}] \\
K_{V}=K_{M} / K_{S}=0.60351572[\mathrm{M} / \mathrm{S}] \\
K_{J}=K_{K g} K_{V}^{2}=9.1531103 E-38[\mathrm{~J}] \text { or } K_{e v}=5.712922471 E-19[\mathrm{ev}]
\end{gathered}
$$

These constants are to be multiplied times the unitless values to obtain measured values in MKS units. Note the similarity of $K_{M}$ and $K_{S}$.

The $K$ 's are the equivalents of the US9 value of " 1 ".

$1 \mathrm{ev}$ is equivalent to a US9 mass: $\underline{M}_{\mathrm{lev}}=7.127827701$

US9 requires minimum and maximum values for all parameters. This is required to prevent information processing in Nature from the infinities in the Standard model.

As energy,

$$
\underline{m}_{e}^{3} / 2=\underline{\hbar} / 2=\operatorname{Spin} 1 / 2=2.381564 E 19
$$

or $\left[\times K_{e v}=13.60569049 \mathrm{ev}\right]$

\section{Mystery of US9-1 Table 2 from US9-1}

In US9-1, Table 2 demonstrated that for 22 fundamental particles the values of $\underline{\Delta t}$ could be represented using only $\underline{m}_{e}$ and @ with coefficients of relatively low value, $1.785 \pm 1.097$. Also, $\underline{M}$ could be represented by $\underline{m}_{e}, R$ and $@, 1.309 \pm$ 0.580 . This clearly indicates that the internal processing of fundamental particles generates particles in terms of US9 constants.

\section{The Uncertainty Principle}

In US9, Einstein's rest energy and Plank's constant are intimately related:

$$
\underline{M} \underline{c}^{2}=R_{M} \underline{m}_{e} \underline{m}_{e}^{2} @^{2}=R_{M} \underline{\hbar} @^{2}=R_{M} \underline{\hbar} /\left[1 / @^{2}\right]
$$

or $R_{M} \underline{\hbar}$ per $1.28808867 E-21 \mathrm{sec}$.

For the electron: $R_{M}=1$.

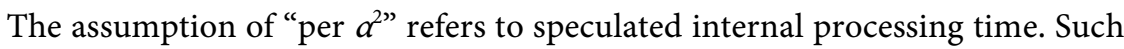
relationships are not possible with MKS units. The association of rest energy with the Uncertainty Principle has a much deeper meaning [4].

$\underline{\Delta}=\underline{m}_{e} \underline{\Delta} v \quad$ assume $\underline{\Delta} x=\underline{\lambda}_{d B} / 2$

In the Bohr Hydrogen atom:

$$
\begin{gathered}
\underline{\lambda}_{d B}=\underline{h} / \underline{m}_{e} \underline{v}=2 \pi \underline{m}_{e}^{3} / \underline{m}_{e}\left[\underline{m}_{e} / n_{B}\right]=n_{B} 2 \pi \underline{m}_{e} \\
\underline{\Delta} \underline{\Delta} x \geq \pm \underline{\hbar} / 2= \pm \underline{m}_{e} \underline{\Delta} \underline{\lambda}_{d B} / 2= \pm m_{e} \underline{\Delta} v n_{B} 2 \pi \underline{m}_{e} / 2= \pm \underline{m}_{e}^{2} \underline{\Delta} v n_{B} \pi \\
\underline{\Delta} v= \pm\left[\underline{m}_{e}^{3} / 2\right] / \underline{m}_{e}^{2} n_{B} \pi= \pm \underline{m}_{e} / n_{B} 2 \pi \\
\underline{\Delta} v / \underline{v}= \pm\left[\underline{m}_{e} / n_{B} 2 \pi\right] /\left[\underline{m}_{e} / n_{B}\right]= \pm 1 / 2 \pi \\
\underline{M} \underline{c}^{2} \underline{\Delta} t_{d B}=R_{M} \underline{m}_{e} \underline{m}_{e}^{2} @{ }^{2} 2 \pi / R_{M} @{ }^{2}=2 \pi \underline{m}_{e}^{3}=\underline{h}= \pm 4 \pi \underline{\Delta E} \underline{\Delta} t= \pm 4 \pi \underline{\Delta} \underline{\Delta} \underline{\Delta}
\end{gathered}
$$

Can the U.P. contain non-random decisions? In order to retain particle identity, avoidance of damaging collisions could be maintained by appropriate digital motion. This could be the justification for the mysterious Uncertainty Principle. 
Even if a small part of the U.P. were systematic, Physics would require adjust-

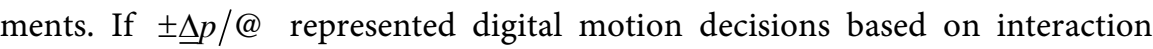
communications, there would be systematic errors in Standard Physics. This will be discussed later in this paper.

\section{Internal Proton Structure}

Force fields originate from particles. An Internal structure is required to produce communication and to react to the communicated field.

The internal structure of the proton is enabled via the elimination of the electrostatic force and the part of the strong force that counters the electrostatic force within the proton. The inefficiency of opposing forces requires excessive information processing that is unlikely in Nature.

Electrostatic force from the proton is inactive over the proton radius. This occurs because the deBroglie communication does not complete a full cycle. The proton radius has been measured to be about $0.86 \mathrm{E}-15$ meters or $/ K_{M}=58.9$. The gravitational force replaces the need for the strong force. Gravitational orbits are possible.

Applying the deBroglie period to the proton:

$$
\underline{\Delta} t_{d B p}=\underline{h} / \underline{M}_{P} \underline{c}^{2}=2 \pi \underline{m}_{e}^{3} / R \underline{m}_{e} \underline{m}_{e}^{2} @^{2}=2 \pi / R @^{2}=1.822225567 E-7
$$

or $\left[\times K_{S}=4.407752875 E-24 \mathrm{sec}\right]$

The time required for a round trip communication which enables definition of distance is

$$
N_{r} 2 \underline{\Delta} t_{d B p}=N_{r} 3.644451134 E-7
$$

or $\left[\times K_{s}=N_{r} 8.81550575 E-24 \mathrm{sec}\right]$

which replaces " $r$ " internally as the measurement of distance between interacting particles.

Minimum Proton electrostatic comm. distance:

$$
\underline{r}_{p}=\underline{\Delta t}_{d \mathrm{~d} p} \underline{\underline{c}}=2 \pi \underline{m}_{e} / R @=90.51785422
$$

or $\left[\times K_{M}=1.321411046 E-15 \mathrm{~m}\right]$

The larger value, $\times 1.54$, requires a new theory regarding the quantized distance force. The similarity of the measured proton radius value and the value from the deBroglie period indicates the following hypothesis.

Hypothesis I: The electrostatic force requires quantized communication between interacting particles to determine distance. Electrostatic force from the proton is inactive over the proton radius. Gravitational internal structure is possible.

For relativistic particles, the distance determined would be significantly over estimated producing a zone of variable reaction to the electrostatic force. This assumes that a return signal is necessary to determine the distance between two interacting particles. The near coincidence between the radius measurement and the deBroglie distance is one justification for this dead zone. The information required to use the strong force to counter the electrostatic force is unnaturally 
inefficient. The chaotic internal state of the proton predicted by the Standard Model is inappropriate for the foundation particle of the Universe. Gravitation may be the foundation of internal particle structure and E\&M may be a later development in the evolution of Physics.

Applying the deBroglie period to the electron:

$$
\underline{\Delta} t_{d B e}=2 \pi / @^{2}=\underline{h} / \underline{m}_{e} \underline{c}^{2}=3.345884344 E-4
$$

or $\left[\times K_{s}=4.747422865 E-21 \mathrm{~s}\right]$

Minimum electron electrostatic comm. distance:

$$
\underline{r}_{e}=\underline{\Delta t} t_{d B e} \underline{C}=2 \pi \underline{m}_{e} / @=1.662044478 E 5
$$

or $\left[\times K_{M}=2.426310203 E-12 \mathrm{~m}\right]$

This is the ground radius of the Hydrogen atom divided by 21.81. Calculation of superconductivity would be altered if an electronic dead zone does occur. This is a quantized distance measurement. This contributes greatly to the Uncertainty Principle.

The time required for a round trip communication which enables definition of distance is

$$
2 \underline{\Delta} t_{d B e}=6.691768688 E-4
$$

or $\left[\times K_{s}=9.49484573 E-21 \mathrm{~s}\right]$

If we assign the deBroglie period to the spin rotation period:

$$
\begin{gathered}
2 \pi \underline{r} / \underline{v}=2 \pi / R_{M} @^{2} \\
\underline{r}=\underline{v} / R_{M} @^{2} \\
\underline{M} \underline{v} \underline{r}=R_{M} \underline{m}_{e} \underline{v}^{2} / R_{M} @^{2}=\underline{m}_{e} \underline{v}^{2} / @^{2}=\underline{\hbar} / 2=\underline{m}_{e}^{3} / 2 \\
\underline{v}^{2}=\underline{m}_{e}^{2} @^{2} / 2=\underline{c}^{2} / 2 \\
\underline{v}= \pm \underline{c} / 2^{1 / 2} \\
\gamma=1 /\left(1-\left[1 / 2^{1 / 2}\right]^{2}\right)^{1 / 2}=3.414213562
\end{gathered}
$$

Spin velocity is independent of mass.

proton: $R_{M}=\gamma R$ electron: $R_{M}=\gamma$

$$
\begin{gathered}
\underline{r}=\underline{v} / R_{M} @^{2} \\
\underline{r}_{e}=\underline{c} / 2^{1 / 2} 3.414213562 @ @^{2}=5.478442307 E 3
\end{gathered}
$$

or $\left[\times K_{M}=7.997620182 E-14 \mathrm{~m}\right]$ or about $0.15 \%$ of the $\mathrm{H}_{1}$ radius.

$$
\underline{r}_{p}=\underline{c} / 2^{1 / 2} 3.414213562 R @^{2}=2.98365234
$$

or $\left[\times K_{M}=4.355640086 E-17 \mathrm{~m}\right]$ or about $3 \%$ of the proton radius.

These values are possible.

Hypothesis II: The proton can be considered as a gravitational binary. 
US9: $\underline{G}=1 / R \underline{m}_{e}^{4}=1 / \underline{M}_{P} \underline{\hbar}=1 / R \underline{e}^{2}$

Kepler's Harmonic Law in US9 for gravitational orbits:

$$
\underline{P}^{2}=4 \pi^{2} \underline{a}^{3} / \underline{G}\left[\underline{M}_{1}+\underline{M}_{2}\right]=4 \pi^{2} \underline{a}^{3} R \underline{m}_{e}^{4} / \underline{M}
$$

$R \underline{m}_{e}=\underline{M}$ is the total mass of a proton binary system.

$$
\begin{gathered}
\underline{P}^{2}=4 \pi^{2} \underline{a}^{3} \underline{m}^{3}=4 \pi^{2} \underline{a}^{3} \underline{\hbar} \\
\underline{a}^{3}=\underline{P}^{2} / 4 \pi^{2} \underline{\underline{m}}_{e}^{3}=\underline{P}^{2} / 4 \pi^{2} \underline{\hbar}= \pm \underline{P}^{2} / 8 \pi^{2} \underline{\Delta} \underline{\underline{\Delta}} x
\end{gathered}
$$

This relationship between the gravitational orbit radius and the Uncertainty Principle is a deeper mystery.

For electron: $\underline{P}^{2}=4 \pi^{2} \underline{a}^{3} R \underline{m}_{e}^{4} / \underline{m}_{e}=4 \pi^{2} \underline{a}^{3} R \underline{m}_{e}^{3}$

For $\underline{a}=1$ or $\left[x K_{M}=1.4598347 E-17 \mathrm{~m}\right]$

$$
\underline{P}_{p}=2 \pi \underline{m}_{e}^{3 / 2}=4.336367035 E 10
$$

or $\left[\times K_{S}=1.048917027 E-6 \mathrm{sec}\right]$

$$
\underline{P}_{e}=2 \pi R^{1 / 2} \underline{m}_{e}^{3 / 2}=1.858148541 E 12
$$

or $\left[\times K_{S}=4.494646389 E-5 \mathrm{sec}\right]$

Electron Gravitational Binaries:

$$
\begin{aligned}
& \underline{P}^{2}=4 \pi^{2} \underline{a}^{3} / \underline{G}_{e}=4 \pi^{2} \underline{a}^{3} R \underline{m}_{e}^{3}=4 \pi^{2} \underline{a}^{3} \underline{\hbar} \\
& \underline{P}=\underline{\Delta}_{d B e}=2 \pi / @^{2}=3.345881282 E-4
\end{aligned}
$$

or $\left[\times K_{S}=8.093299804 E-21 \mathrm{sec}\right]$

$$
\begin{gathered}
\underline{P}^{2}=4 \pi^{2} / @^{4}=4 \pi^{2} \underline{a}^{3} R \underline{m}_{e}^{3} \\
\underline{a}^{3}=1 / R \underline{m}_{e}^{3} @^{4}=1 / R \underline{\hbar} @^{4} \\
\underline{a}=1 / R^{1 / 3} \underline{m}_{e} @^{4 / 3}=3.188747033 E-11
\end{gathered}
$$

or $\left[\times K_{M}=4.655043568 E-28 \mathrm{~m}\right]$

This result is possible.

Hypothesis III: All known fundamental particles can be considered as gravitational binaries.

Assuming that the lifetimes of all fundamental particles are orbital periods yields reasonable gravitational orbits down to periods of $1 \mathrm{E}-25$ seconds. This is demonstrated in Table 1 [5].

Calculations for Table 1 and Table 2:

$$
\underline{P}^{2}=4 \pi^{2} \underline{a}^{3} / \underline{G}\left(\underline{M}_{1}+\underline{M}_{2}\right)=4 \pi^{2} \underline{a}^{3} R \underline{m}_{e}^{4} / \underline{M}
$$

assume $\underline{P}=\underline{\Delta} t=\Delta t \mathrm{sec} / 2.41888433 E-17=$ lifetime

$$
\begin{gathered}
\underline{a}=\left(\underline{\Delta t} \underline{M} / 4 \pi^{2} R \underline{m}_{e}^{4}\right)^{1 / 3}=\underline{\Delta t^{2 / 3}} M^{1 / 3} / 2.32177175 E 10 \\
\underline{v}=2 \pi \underline{a} / \underline{\Delta} t
\end{gathered}
$$

The velocity in US9 units is only 1.657 larger than in MKS units.

Values for the electron and proton are estimated via $\underline{a}=1$. 
Table 1. Gravitational Orbits for 22 fundamental particles in US9.

\begin{tabular}{|c|c|c|c|c|c|c|}
\hline$\Delta \mathrm{t}[\mathrm{sec}]$ & $\underline{V}$ & $\underline{\Delta t}$ & $\underline{M}$ & Particle & $\underline{a}$ & $\underline{a}$ (US9) \\
\hline $4.4946 \mathrm{E}-5$ & $3.3817 \mathrm{E}-12$ & $1.858 \mathrm{E} 12$ & $3.625 \mathrm{E} 6$ & e & 1 & 1 \\
\hline $1.0489 \mathrm{E}-6$ & $1.4490 \mathrm{E}-10$ & $4.336 \mathrm{E} 10$ & $6.655 \mathrm{E} 9$ & $\mathrm{p}$ uud & 1 & 1 \\
\hline $2.2 \mathrm{E}-6$ & $5.4664 \mathrm{E}-11$ & $9.10 \mathrm{E} 10$ & $7.50 \mathrm{E} 8$ & $\mu$ & $7.9170 \mathrm{E}-1$ & 0.7917 \\
\hline $5.12 \mathrm{E}-8$ & $3.2093 \mathrm{E}-10$ & 2.117E9 & $3.531 \mathrm{E} 9$ & $K_{L}^{0} \quad \mathrm{~d} \underline{\mathrm{s}}+$ & $1.0813 \mathrm{E}-1$ & 0.1081 \\
\hline $2.603 \mathrm{E}-8$ & $2.6336 \mathrm{E}-10$ & $1.076 \mathrm{E} 9$ & $9.90078 \mathrm{E} 8$ & $\pi^{+-}$ud & $4.5076 \mathrm{E}-2$ & $6.180 @^{-1}$ \\
\hline $1.24 \mathrm{E}-8$ & $5.2462 \mathrm{E}-10$ & $5.126 \mathrm{E} 8$ & $3.50 \mathrm{E} 9$ & $\mathrm{~K}^{+} \mathrm{us}$ & $4.1885 \mathrm{E}-2$ & $5.865 @^{-1}$ \\
\hline $2.9 \mathrm{E}-10$ & $2.4864 \mathrm{E}-9$ & $1.199 \mathrm{E} 7$ & 9.3E9 & $\Xi^{0}$ uss & $4.7448 \mathrm{E}-3$ & $0.650 @^{-1}$ \\
\hline $2.63 \mathrm{E}-10$ & $2.4347 \mathrm{E}-9$ & $1.087 \mathrm{E} 7$ & 7.915 E9 & $\Lambda^{0}$ uds & $4.2120 \mathrm{E}-3$ & $0.577 @^{-1}$ \\
\hline $1.64 \mathrm{E}-10$ & $3.015 \mathrm{E}-9$ & $6.78 \mathrm{E} 6$ & $9.376 \mathrm{E} 9$ & $\Xi^{-} \mathrm{dss}$ & $3.2534 \mathrm{E}-3$ & $0.466 @^{-1}$ \\
\hline $1.48 \mathrm{E}-10$ & $3.019 \mathrm{E}-9$ & $6.12 \mathrm{E} 6$ & $8.4941 \mathrm{E} 9$ & $\Sigma^{-} \mathrm{dds}$ & $2.9403 \mathrm{E}-3$ & $0.403 @^{-1}$ \\
\hline $0.895 \mathrm{E}-10$ & $2.664 \mathrm{E}-9$ & $3.70 \mathrm{E} 6$ & $3.530 \mathrm{E} 9$ & $K_{s}^{0} \quad$ ds- & $1.5688 \mathrm{E}-3$ & $0.215 @^{-1}$ \\
\hline $0.82 \mathrm{E}-10$ & $4.109 \mathrm{E}-9$ & 3.39E6 & $1.1864 \mathrm{E} 10$ & $\Omega^{-} s s b$ & $2.2168 \mathrm{E}-3$ & $0.304 @^{-1}$ \\
\hline $0.80 \mathrm{E}-10$ & $3.697 \mathrm{E}-9$ & $3.31 \mathrm{E} 6$ & $8.4373 \mathrm{E} 9$ & $\Sigma^{+}$uus & $1.9475 \mathrm{E}-3$ & $0.267 @^{-1}$ \\
\hline $1.04 \mathrm{E}-12$ & $1.828 \mathrm{E}-8$ & $4.30 \mathrm{E} 4$ & $1.3263 \mathrm{E} 10$ & $\mathrm{D}^{+} \mathrm{cd}$ & $1.2513 \mathrm{E}-4$ & $2.35 @^{-2}$ \\
\hline $4.10 \mathrm{E}-13$ & $2.494 \mathrm{E}-8$ & $1.69 \mathrm{E} 4$ & $1.3228 \mathrm{E} 10$ & $\mathrm{D}^{0} \mathrm{cu}$ & $6.7082 \mathrm{E}-5$ & $1.26 @ @^{-2}$ \\
\hline $3.2 \mathrm{E}-13$ & $2.672 \mathrm{E}-8$ & $1.32 \mathrm{E} 4$ & $1.27 \mathrm{E} 10$ & $\mathrm{~T}^{-}$ & $5.6126 \mathrm{E}-5$ & $1.05 @^{-2}$ \\
\hline $2.0 \mathrm{E}-13$ & $3.387 \mathrm{E}-8$ & $8.27 \mathrm{E} 3$ & $1.622 \mathrm{E} 10$ & $\Lambda_{\mathrm{c}}^{+} \mathrm{udc}$ & $4.4586 \mathrm{E}-5$ & $1.05 @^{-2}$ \\
\hline $8.5 \mathrm{E}-17$ & $1.757 \mathrm{E}-7$ & 3.51 & $9.57638 \mathrm{E} 8$ & $\pi^{0}$ uu - & $9.8126 \mathrm{E}-8$ & $0.252 @^{-3}$ \\
\hline $5.0 \mathrm{E}-19$ & $1.550 \mathrm{E}-6$ & $2.07 \mathrm{E}-2$ & 3.8864E9 & $\eta$ uu + & $5.1052 \mathrm{E}-9$ & $1.80 @^{-4}$ \\
\hline $0.74 \mathrm{E}-19$ & $3.798 \mathrm{E}-6$ & $3.06 \mathrm{E}-3$ & $8.4600 \mathrm{E} 9$ & $\Sigma^{0}$ uds & $1.8498 \mathrm{E}-9$ & $0.652 @^{-4}$ \\
\hline $1 \mathrm{E}-25$ & $1.402 \mathrm{E}-3$ & $4.1 \mathrm{E}-9$ & $5.7 \mathrm{E} 11$ & $\mathrm{~W}$ & $9.1480 \mathrm{E}-13$ & $6.06 @^{-6}$ \\
\hline $1 \mathrm{E}-25$ & $1.465 \mathrm{E}-3$ & $4.1 \mathrm{E}-9$ & $6.5 \mathrm{E} 11$ & $\mathrm{Z}_{0}$ & $9.5574 \mathrm{E}-13$ & $6.33 @^{-6}$ \\
\hline
\end{tabular}

\section{Conclusions from the Gravitational Orbit Table}

Values of " $\underline{\text { " [ }} E-17$ to $E-29 \mathrm{~m}$ ] fit deep within the dead zone postulated for electrostatics. The value of $\underline{G}$ seems tailored for this purpose.

Hypothesis IV: All binary components of fundamental particles are also gravitational binaries.

There would be $2^{\mathrm{n}}$ components associated with each fundamental particle. Perhaps some of these are quarks. Here, assume a proton binary.

$$
\begin{gathered}
\underline{P}^{2}=4 \pi^{2} \underline{a}^{3} / \underline{G R} \underline{m}_{e}=4 \pi^{2} \underline{a}^{3} \underline{m}_{e}^{3} \\
2^{22}=1.157077532 \underline{m}_{e} \\
\underline{m}_{e}=10 ! / 1.0010726=2^{22} / 0.8642463207=3.6249118 E 6 \\
\underline{P}_{n}=2 \pi \underline{a}^{3 / 2} \underline{\underline{m}}_{e}^{3 / 2} 2^{[n-1]] / 2}
\end{gathered}
$$

“a” must decrease to avoid orbital interactions.let $\underline{a}=1 / 4^{n-1}$

$$
\begin{aligned}
\underline{a}^{3 / 2} & =1 / 4^{[n-1] 3 / 2} \quad \underline{P}_{n}=\left[2 \pi \underline{m}_{e}^{3 / 2}\right] 2^{[n-1] 1 / 2} / 4^{[n-1] 3 / 2} \\
\underline{P}_{n} & =4.3363677035 E 10\left[2^{[n-1] / 2} / 4^{[n-1] 3 / 2}\right]
\end{aligned}
$$


Table 2. Gravitational Binary Orbits for $4.196 E 6$ particle system in US9.

\begin{tabular}{|c|c|c|c|c|c|c|}
\hline$\underline{p}$ US 9 & $\mathrm{P}[\mathrm{sec}]$ & Particle Total & & Particle Mass Ratio & $\underline{a}$ & a meter \\
\hline $4.336 \mathrm{E} 10$ & $1.049 \mathrm{E}-6$ & 2 & & $\mathrm{p}$ & 1 & $1.456 \mathrm{E}-17$ \\
\hline $7.666 \mathrm{E} 9$ & $1.855 \mathrm{E}-7$ & 4 & 2 & $1 / 2$ & $2.500 \mathrm{E}-1$ & $3.640 \mathrm{E}-18$ \\
\hline $2.710 \mathrm{E} 9$ & $6.555 \mathrm{E}-8$ & 8 & 3 & $1 / 4$ & $6.250 \mathrm{E}-2$ & $9.100 \mathrm{E}-19$ \\
\hline $2.396 \mathrm{E} 8$ & $5.796 \mathrm{E}-9$ & 16 & 4 & $1 / 8$ & $1.563 \mathrm{E}-2$ & $2.275 \mathrm{E}-19$ \\
\hline $4.235 \mathrm{E} 7$ & $1.024 \mathrm{E}-9$ & 32 & 5 & $1 / 16$ & $3.906 \mathrm{E}-3$ & $5.688 \mathrm{E}-20$ \\
\hline $6.234 \mathrm{E} 6$ & $1.508 \mathrm{E}-10$ & 64 & 6 & $1 / 32$ & $9.766 \mathrm{E}-4$ & $1.422 \mathrm{E}-20$ \\
\hline $1.666 \mathrm{E} 6$ & $4.030 \mathrm{E}-11$ & 128 & 7 & $1 / 64$ & $2.441 \mathrm{E}-4$ & $3.555 \mathrm{E}-21$ \\
\hline $2.339 \mathrm{E} 5$ & $5.658 \mathrm{E}-12$ & 256 & 8 & $1 / 128$ & $6.104 \mathrm{E}-5$ & $8.887 \mathrm{E}-22$ \\
\hline $4.135 \mathrm{E} 4$ & $1.000 \mathrm{E}-12$ & 512 & 9 & $1 / 256$ & $1.526 \mathrm{E}-5$ & $2.222 \mathrm{E}-22$ \\
\hline $7.311 \mathrm{E} 3$ & $1.768 \mathrm{E}-13$ & 1024 & 10 & $1 / 512$ & $3.815 \mathrm{E}-6$ & $5.554 \mathrm{E}-23$ \\
\hline $1.292 \mathrm{E} 3$ & $3.125 \mathrm{E}-14$ & 2048 & 11 & $1 / 1024$ & $9.538 \mathrm{E}-7$ & $1.389 \mathrm{E}-23$ \\
\hline $2.285 \mathrm{E} 2$ & $5.526 \mathrm{E}-15$ & 4096 & 12 & $1 / 2048$ & $2.384 \mathrm{E}-7$ & $3.471 \mathrm{E}-24$ \\
\hline $4.039 \mathrm{E} 1$ & $9.770 \mathrm{E}-16$ & 8192 & 13 & $1 / 4096$ & $5.961 \mathrm{E}-8$ & $8.678 \mathrm{E}-25$ \\
\hline 7.139 & $1.727 \mathrm{E}-16$ & 16384 & 14 & $1 / 8192$ & $1.490 \mathrm{E}-8$ & $2.170 \mathrm{E}-25$ \\
\hline 1.262 & $3.053 \mathrm{E}-17$ & 32768 & 15 & $2.441 \mathrm{E}-4$ & $3.726 \mathrm{E}-9$ & $5.424 \mathrm{E}-26$ \\
\hline $2.231 \mathrm{E}-1$ & $5.397 \mathrm{E}-18$ & 65536 & 16 & $1.221 \mathrm{E}-4$ & $9.314 \mathrm{E}-10$ & $1.356 \mathrm{E}-26$ \\
\hline $3.944 \mathrm{E}-2$ & $9.540 \mathrm{E}-19$ & 131072 & 17 & $6.105 \mathrm{E}-5$ & $2.328 \mathrm{E}-10$ & $3.390 \mathrm{E}-27$ \\
\hline $6.972 \mathrm{E}-3$ & $1.686 \mathrm{E}-19$ & 262144 & 18 & $3.053 \mathrm{E}-5$ & $5.821 \mathrm{E}-11$ & $8.475 \mathrm{E}-28$ \\
\hline $1.232 \mathrm{E}-3$ & $2.980 \mathrm{E}-20$ & 524288 & 19 & $1.526 \mathrm{E}-5$ & $1.455 \mathrm{E}-11$ & $2.119 \mathrm{E}-28$ \\
\hline $2.179 \mathrm{E}-4$ & $5.271 \mathrm{E}-21$ & 1048576 & 20 & $7.631 \mathrm{E}-6$ & $3.638 \mathrm{E}-12$ & $5.297 \mathrm{E}-29$ \\
\hline $3.851 \mathrm{E}-5$ & $9.315 \mathrm{E}-22$ & 2097152 & 21 & $3.816 \mathrm{E}-6$ & $9.095 \mathrm{E}-13$ & $1.324 \mathrm{E}-29$ \\
\hline $6.808 \mathrm{E}-6$ & $1.647 \mathrm{E}-22$ & 4194304 & 22 & $1.908 \mathrm{E}-6$ & $2.274 \mathrm{E}-13$ & $3.310 \mathrm{E}-30$ \\
\hline
\end{tabular}

\section{Conclusions:}

This particular model nearly covers the range of periods in Table 1 . The proton binary can be composed of two distinct systems which communicate and interact. To relate with the quark model, we could introduce a triplet.

Table 2 expresses a potential for sufficient complexity in the proton to generate the Laws of Physics. Needed is a rationale for a minimum value of distance. This type of complexity along with internal communication structure is a requirement for particle generation of the fields of the Standard Model and for particle reaction to those fields.

Efficient information processing is the fundamental requirement for the maintenance of particle lifetime. Nature is governed by internal processing. This enables evolution to produce atoms, molecules, biology and higher life forms. Ignoring this fact has resulted in the stagnation of the Standard Model.

The primary binary can form two or three interacting information systems which act as a fundamental brain. Internal communication can be via gravitons 
which impart energy to the appropriate orbits in $+/-$ momentum changes. Defining the Uncertainty Principle as the Organizational Principle would increase understanding of internal mechanisms. Finding the mode that enables evolution would enlighten biological studies, because the fundamentals of evolution are preserved in all entities.

\section{Define Digital Parameters at Nonrelativistic Velocities}

Hypothesis V: Fundamental particle dynamics arise from internal digital decisions based on an internal clock which is coordinated with external cosmic and local communications.

The following presents a digital unitless physics in which all velocities occur due to internal processing delay. The time interval for all physical processes is a US9 constant, $[1 / @]=[\alpha]$, independent of velocity and mass.

$$
\Delta t_{0}=1 / @
$$

or $\left[\times K_{S}=1.76514518 E-19 \mathrm{sec}\right]$

Define digital parameters at nonrelativistic velocities:

$$
\begin{gathered}
\underline{\Delta} t_{0}=1 / @ \quad N_{c}=\underline{m}_{e} / \underline{\delta} x_{M} \\
N_{c}=R_{M} \underline{m}_{e}^{2} @=\underline{M} \underline{c} \underline{\delta} x_{M}=\underline{m}_{e} / N_{c}=R_{M} \underline{m} @=1 / \underline{M} @ \\
\underline{\Delta} t_{0} / N_{c}=\alpha / R_{M} \underline{m}_{e}^{2} @=1 / R_{M} \underline{m}_{e}^{2} @^{2}=1 / R_{M} \underline{c}^{2}
\end{gathered}
$$

For electron: $R_{M}=1$

$$
\underline{\Delta} t_{0} / N_{c e}=1 / \underline{m}_{e}^{2} @^{2}=1 / \underline{c}^{2}=4.052618953 E-18
$$

or $\left[\times K_{S}=9.802816481 E-35 \mathrm{sec}\right]$, time per electron step

$$
\underline{\delta}_{e}=1 / \underline{m}_{e} @=2.013111759 E-9
$$

or $\left[\times K_{M}=2.9388104 E-26 \mathrm{M}\right]$ step size

$$
\underline{\delta} t_{e}=\underline{\delta} x_{e} / \underline{c}=1 / \underline{c}^{2}=4.052618953 E-18
$$

or $\left[\times K_{s}=9.80281648 E-35 \mathrm{sec}\right]$ step time

$N_{c} \underline{\delta} x_{M}=\underline{m}_{e}$ maximum distance per $\alpha$

For proton:

$$
R_{M}=1836.152672 \underline{\Delta t} / N_{c p}=1 / R \underline{m}_{e}^{2} @^{2}=2.20712565 E-21
$$

or $\left[\times K_{S}=5.338780718 E-38 \mathrm{sec}\right]$ time per proton step

$$
\underline{\delta} x_{P}=1 / R \underline{m}_{e} @=1.096374931 E-12
$$

or $\left[\times K_{M}=1.600526168 E-29 \mathrm{M}\right]$ proton step size

$$
\underline{\delta} t_{P}=\underline{\delta} x_{P} / \underline{c}=1 / R \underline{c}^{2}=2.207125265 E-21
$$

or $\left[\times K_{s}=5.338780718 E-38 \mathrm{sec}\right]$

$N_{c} \underline{\delta} x_{P}=\underline{m}_{e}$ maximum distance at velocity $\underline{c}$ per $\alpha$

$\underline{c}_{d B p} / \underline{\delta} x_{M}=\left[\underline{m}_{e} @\right]\left[2 \pi / R_{M} @^{2}\right]\left[R_{M} \underline{m}_{e} @\right]=2 \pi \underline{m}_{e}^{2}=2 \pi \underline{e}=8.256096419 E 13$

\section{Define velocity}




$$
N_{v}=\beta N_{c}=\beta \underline{M} \underline{c}=\underline{M} \underline{v}=\underline{p} \underline{\begin{array}{c}
\text { in increments of } 1 \\
\underline{v}_{\min }=1 / R_{M} \underline{m}_{e}
\end{array}}
$$

This results in quantized momentum at a low level, $4.030 \mathrm{E}-37 \mathrm{~kg} \cdot \mathrm{m} / \mathrm{s}$.

$$
\begin{gathered}
\underline{\lambda}_{d B}=\underline{h} / \underline{M} \underline{v}=2 \pi \underline{m}_{e}^{3} / N_{v} \quad N_{v} \underline{\lambda}_{d B}=\underline{h} \\
N_{v} \underline{\lambda}_{d B}=\underline{h}
\end{gathered}
$$

Bohr atom:

$$
\begin{gathered}
\underline{r}_{n}=n^{2} \underline{m}_{e} \quad \underline{v}_{n}=\underline{m}_{e} / n \quad \underline{p}_{n}=\underline{m}_{e}^{2} / n=N_{v} \\
N_{v} n=\underline{m}_{e}^{2}=\underline{e} \quad \underline{\lambda}=4 \pi \underline{m}_{e} @ n_{f}^{2} n_{i}^{2} /\left[n_{f}^{2}-n_{i}^{2}\right]
\end{gathered}
$$

For $n_{f}=2, n_{i}=1$ :

$$
\underline{\lambda}=4 \pi \underline{m}_{e} @ 4 / 3=8.323015723 E 9
$$

or $\left[\times K_{M}=1.215022716 E-7 \mathrm{~m}\right] 1215 \mathrm{Ang}$

The critical factor in this approach is that motion occurs digitally after communication interaction with other particles. It also includes internal information processing delays which result in the velocity of light and all lower velocities. It further allows the possibility that internal processing may result in negative motion that promotes higher organization (creativity). Adjusting motion to avoid collisions may be the source of the Uncertainty Principle.

Introducing an internal perspective on Special Relativity:

Hypothesis VI: SR gamma is the result of a limitation on internal temporal data.

time dilation in S.R.:

$$
\begin{gathered}
\Delta t_{\text {meas }}=\gamma \Delta t_{\text {rest }}=\Delta t_{\text {rest }} /\left(1-\beta^{2}\right)^{1 / 2} \\
\Delta t_{\text {meas }}\left(1-\beta^{2}\right)^{1 / 2}=\Delta t_{\text {rest }}
\end{gathered}
$$

squaring this:

$$
\begin{gathered}
\Delta t_{\text {meas }}^{2}=\beta^{2} \Delta t_{\text {meas }}^{2}+\Delta t_{\text {rest }}^{2}=\left[v^{2} \Delta t_{\text {meas }}^{2} / c^{2}\right]+\Delta t_{\text {rest }}^{2} \\
{\left[\underline{v}^{2} \underline{\Delta} t_{\text {meas }}^{2} / \underline{c}^{2}\right]=\underline{\Delta} r^{2} / \underline{c}^{2}=N_{v}^{2} \underline{\delta} x_{M}^{2} / \underline{c}^{2}=N_{v}^{2} \underline{\delta} t_{M}^{2}}
\end{gathered}
$$

This term is obviously an orthogonal delay proportional to distance traveled. Internal digital motion step processing delay can replace the external space-time concept.

Electrostatic Acceleration from proton on electron:

$$
\begin{gathered}
\underline{\Delta} v_{E}=\underline{\Delta t \gamma} \underline{m}_{e}^{2} / \underline{r}^{2}=\alpha \gamma \underline{\hbar} / \underline{r}^{2} \\
\underline{\Delta} x_{E}=\underline{\Delta} t^{2} \gamma^{2} \underline{m}_{e}^{3} / \underline{r}^{2}=\alpha^{2}\left[1-\beta^{2}\right] \underline{\hbar} / \underline{r}^{2} \underline{r}^{2}=\underline{\Delta} t_{r}^{2} \underline{c} \underline{c}^{2}=\Delta t_{r} \underline{m}_{e}^{2} @{ }^{2}
\end{gathered}
$$

The association of $\underline{\hbar}$ with acceleration is another intriguing mystery.

The U.P. association makes excessive acceleration possible, and perhaps eliminates the dark matter requirement. The distance, $\underline{r}$, is determined via communication becoming an internal time parameter. 


\section{Communication Theory}

The assumptions of the Standard Model of Physics do not allow generation of many of the known facts of Physics. Primary among these are the velocity of light, the gravitational force, the electrostatic forces, Mach's principle, inertia and alocality in quantum mechanics. Although the Standard Model provides adequate description of these facts, it does not provide the possibility of generation of the bases of physical law. This is primarily because communication is not allowed to exceed the velocity of light and arbitrary units do not provide sufficient insight [6].

Therefore, we postulate two communication velocities that exceed the velocity of light for consideration under the US9 unitless system.

Hypothesis VII: The velocities $\underline{c}^{2}$ and $\underline{c}^{3}$ are required communication velocities for the interaction of fundamental particles which enables the U.P. to create greater complexity.

Communication between particles requires both local and cosmic interactions. In US9 it is simplest to consider $\underline{c} \underline{c}^{2}$ and $\underline{c}^{3}$ as communication velocities. Being unitless allows consideration of any of these as communication velocities and $\underline{c}$ is the foundation of Standard Physics.

Three communication velocities for the proton deBroglie period:

$$
\begin{aligned}
\underline{c} \underline{\Delta} t_{d B} & =2 \pi \underline{m}_{e} / R @=\underline{h} / M_{P} \underline{c}=0.660540696 @ \\
\text { or } \times K_{M} & =1.321409837 E-15 \mathrm{~m}
\end{aligned}
$$

$\mathrm{E} \& \mathrm{M}$ is enabled,

$$
\underline{c}^{2} \underline{\Delta t} \underline{t}_{d B}=2 \pi \underline{m}_{e}^{2} / R=\underline{h} / \underline{M}_{P}=2 \pi 1.075176754 R \underline{m}_{e}=4.496410645 E 10
$$

or $\times K_{M}=6.564016285 E-7 \mathrm{~m}$

Gravitational aberration is significantly reduced and spin alocality is enabled. Spin is a gravitational phenomenon. " $\underline{c}$ " is regulated by " $\underline{c}$ " via receptor communication.

$$
\underline{c}^{3} \underline{\Delta} t_{d B}=2 \pi \underline{m}_{e}^{3} @ / R=\underline{h c} / \underline{M}_{P}=2.233562357 E 19
$$

or $\times K_{M}=326.0631833 \mathrm{~m}$

Mach's Principle is enabled. Momentum is defined.

Hypothesis VIII: The gravitational field is generated at velocity $\underline{c}^{2}$ [7].

Particle to particle communication is via gravitinos. The virtual particle scenario is actually internal particle temporal processing.

Gravitational Acceleration from proton on electron:

$$
\underline{G}=1 / R \underline{m}_{e}^{4} \quad \underline{\Delta} t=1 / @=\alpha
$$

Gravitational comm. velocity at $\underline{c}^{2}$ :

$$
\begin{gathered}
\underline{\Delta} v_{G}=\underline{\Delta} t \gamma R \underline{m}_{e} / R \underline{m}_{e}^{4} \underline{r}^{2}=\underline{\Delta} t \gamma / m_{e}^{3} \underline{r}^{2}=\alpha \gamma / \underline{\hbar r}^{2} \\
\underline{\Delta} x_{G}=\underline{\Delta} t^{2} \gamma^{2} / \underline{m}_{e}^{3} \underline{r}^{2}=\alpha^{2}\left[1-\beta^{2}\right] / \underline{\hbar r^{2}} \quad \underline{r}^{2}=\underline{\Delta t} t_{r 2}^{2} \underline{c}^{4} \\
\underline{\Delta x} x_{G}=\alpha^{2} \gamma^{2} / \underline{m}_{e}^{3} \underline{m}^{4} @{ }^{4} \underline{\Delta} t_{r 2}^{2}=\alpha^{6}\left[1-\beta^{2}\right] / \underline{m}_{e}^{7} \underline{\Delta} t_{r 2}^{2}
\end{gathered}
$$

Universe Radius: 1.306E26 [m] observed 
US9: $\underline{U R}=8.89 E 42=0.2087 @^{2} \underline{m}_{e}^{6}=2.134 \underline{e} / \underline{G}=2.134 R \underline{\hbar^{2}}$

Distance to Galactic Center: 26,000 light years:

US9: $\underline{D} \underline{G} \underline{C}=1.685 E 37=1.434 @^{2} \underline{m}_{e}^{5}$

$$
\underline{D} \underline{G} \underline{C} / \underline{c}^{2}=1.434 \underline{m}_{e}^{5} / \underline{m}_{e}^{2}=1.434 \underline{m}_{e}^{3}=1.434 \underline{\hbar}=4.763 E 19
$$

or $\times K_{s}=1152 \mathrm{sec}$

$$
\underline{D} \underline{G} \underline{C} / \underline{c}^{3}=1.434 @^{2} \underline{m}_{e}^{5} / \underline{m}_{e}^{3} @^{3}=1.434 \underline{m}_{e}^{2} / @=1.375 E 11
$$

or $\times K_{s}=3.326 E-6 \mathrm{sec}$ This is near the muon lifetime.

$$
\underline{U R} / \underline{c}^{3}=2.134 R \underline{m}_{e}^{6} / \underline{m}_{e}^{3} @^{3}=2.134 R \underline{m}_{e}^{3} / @^{3}=7.253 E 16
$$

or $\times K_{S}=1.754 \mathrm{sec}$

Hypothesis IX: Inertia, Mach's Principle, rest energy and velocity definition are defined by cosmic communication at velocity $\underline{c}^{3}$.

Inertia, rest energy and velocity definition via cosmic communication is enabled, solving Einstein's dilemma. A particle's identity is defined by mass and velocity with respect to a cosmic frame. The evolution of particle communication probably occurred in the sequence: $\underline{c}^{3}, \underline{c}^{2}, \underline{c}$. Momentum defined, followed by gravitational force, followed by E \& M. This is evolutionary Physics which provides the fundamental properties of biological evolution providing a Universe with increasing information content and increasing complexity of fundamental components.

Gravitational communication inside fundamental particles is now assumed to be at velocity $\underline{c}^{2}$. Internal proton forces require interaction between internal particles. Interaction requires transfer of particle identity and energy transfer.

$$
\underline{\lambda}_{d B}=\underline{c}^{2} / \underline{v} \omega_{d B}=\underline{c}^{2} \underline{P}_{d B} / \underline{v}=\underline{m}_{e}^{2} \varrho^{2} 2 \pi / R_{M} @^{2} \beta \underline{m}_{e} @=2 \pi \underline{m}_{e} / R_{M} \beta @
$$

$$
\text { in H ground orbit } R_{M}=1 \quad \beta=1 / @ \quad \underline{\lambda}_{d B}=2 \pi \underline{m}_{e}
$$

Hypothesis X: Angular Momentum Conservation is the result of gravitational communication delay time.

In an organized system, the radial communication delay time can be assumed to be:

$$
\underline{\Delta} t_{r}=\underline{r} / \underline{c}^{2}=\underline{r} / \underline{m}_{e}^{2} @^{2}
$$

If this is assumed to be the internal digital step time interval:

$$
\begin{gathered}
\underline{v}=N_{v} \underline{\delta}_{e} / \underline{\Delta} t_{r}=N_{v} / R_{M} \underline{m}_{e} \underline{\Delta} t_{r} \\
\underline{M} \underline{v} \underline{r}=R_{M} \underline{m}_{e}\left[N_{v} / R_{M} \underline{m}_{e} \underline{\Delta} t_{r}\right] \underline{c}^{2} \underline{\Delta} t_{r}=N_{v} \underline{c}^{2}=\underline{L}
\end{gathered}
$$

Reduction of $\Delta t_{r}$ results in corresponding digital increase in motion per time.

The law of angular momentum conservation has been used as a basis for much of physics without producing a rationale for the physics which produces the law. Radial communication time is the rationale. " $\underline{c}$ " has been introduced as the gravitational signal velocity communicated along the radius within the suggested internal time, $\alpha$. As " $r$ " is reduced, $\Delta t_{r}$ is reduced which decreases the 
time between steps, $\underline{\delta t}$, producing larger $\underline{\Delta x}$ per time, $\alpha$.

As an example, a tornado would follow this law. The ability to confuse this signal would disrupt the structure of the tornado. $1 \mathrm{E} 3 \mathrm{~m}$ tornado radius $\times 2$ converts in US9 to:

$$
\begin{aligned}
2 \underline{r} & =1.370018126 E 202 \underline{r} / \underline{c}^{2}=555.2161423 \text { or } \\
\times K_{s} & =8.105237905 E-15 \mathrm{sec}
\end{aligned}
$$

Conservation of helicity is angular momentum conservation within fluids. This proves that organized particle to particle communication is well established in Nature.

$\begin{array}{lll}\text { Quarks } & \text { Mass } & \text { Charge } \\ \mathrm{u} & 5.87 \underline{m}_{e} & 2 / 3 \\ \mathrm{~d} & 11.7 \underline{m}_{e} & -1 / 3 \\ \mathrm{~s} & 1.57 @ \underline{m}_{e} & -1 / 3 \\ \mathrm{c} & 1.39 R \underline{m}_{e} & 2 / 3 \\ \mathrm{~b} & 4.48 R \underline{m}_{e} & -1 / 3 \\ \mathrm{t} & 1.40 @ R \underline{m}_{e} & 2 / 3\end{array}$

The more massive group is close to $R \mathrm{x}$ the first group [8].

\section{Neutrinos}

$\underline{M}=R_{M} \underline{m}_{e} \quad$ Values of electron neutrinos mass can be around " 1 ”.

$R_{M}$ includes gamma. $0.139707191 \mathrm{ev} / K_{e v}=1=\underline{M}$ Neutrinos may be gravitinos.

\section{Photons in US9}

Photon view:

$$
\begin{gathered}
\underline{\omega}_{\lambda}=\underline{c} / \underline{\lambda}=\underline{m}_{e} @ / \underline{\lambda} \\
\underline{h}_{\lambda}=\underline{m}_{\lambda} \underline{c}^{2}=\underline{m}_{\lambda} \underline{m}_{e}^{2} @^{2} \\
\underline{\omega}_{\lambda}=\underline{m}_{\lambda} \underline{m}_{e}^{2} @^{2} / 2 \pi \underline{m}_{e}^{3}=\underline{m}_{\lambda} @^{2} / 2 \pi \underline{m}_{e}=R_{\lambda} @^{2} / 2 \pi=1 / P_{\lambda} \\
\underline{P}_{\lambda}=2 \pi / R_{\lambda} @^{2}=P_{d B}
\end{gathered}
$$

The photon mass can be treated as a normal mass.

Wavelength is the Bohr base orbit circumference divided by @ $R_{\lambda}$.

US9 enables new relationships.

$$
\underline{\lambda}_{1}=2 \pi \underline{m}_{e} / R_{1} @
$$

U.P. view: $\underline{\Delta} t_{\lambda}= \pm \underline{P}_{\lambda}= \pm 2 \pi / R_{\lambda} @{ }^{2}= \pm \underline{\lambda} / \underline{c}$

$$
\begin{gathered}
\underline{\Delta E}_{\lambda} \underline{\Delta t}_{\lambda} \geq \pm \underline{\hbar} / 2 \\
\underline{\Delta} E_{\lambda}= \pm \underline{m}_{e}^{3} R_{\lambda} @{ }^{2} / 4 \pi=R_{\lambda} \underline{m}_{e} \underline{c}^{2} / 4 \pi=\underline{m}_{\lambda} \underline{c}^{2} / 4 \pi=\underline{h} \underline{\omega}_{\lambda} / 4 \pi \\
\underline{\Delta p} \underline{\Delta} x=R_{\lambda} \underline{m}_{e} \underline{\Delta} \underline{\underline{\lambda}} / 2= \pm \underline{\hbar} / 2= \pm \underline{m}_{e}^{3} / 2 \\
\underline{\Delta} v= \pm \underline{m}_{e}^{2} / R_{\lambda} \underline{\lambda} \\
\underline{\Delta v} / \underline{c}= \pm \underline{m}_{e} / @ R_{\lambda} \underline{\lambda}= \pm \underline{m}_{e} R_{\lambda} @ / @ R_{\lambda} 2 \pi \underline{m}_{e}= \pm 1 / 2 \pi
\end{gathered}
$$

Does the U.P. apply to photons?

\section{Photons as gravitational binaries:}


Kepler's Harmonic Law Photon Gravitational Binary with $\underline{P}_{\lambda}$ as orbital period [9]

$$
\begin{gathered}
\underline{P}_{\lambda}^{2}=4 \pi^{2} \underline{a}^{3} / \underline{G}\left[\underline{M}_{1}+\underline{M}_{2}\right]=4 \pi^{2} \underline{a}^{3} R \underline{m}_{e}^{4} / \underline{m}_{\lambda} \\
\underline{m}_{\lambda}=R_{\lambda} \underline{m}_{e} \\
\underline{P}_{\lambda}^{2}=4 \pi^{2} \underline{a}^{3} R \underline{m}_{e}^{3} / R_{\lambda}=4 \pi^{2} / R_{\lambda}^{2} @^{4} \\
\underline{a}^{3}=\left[R_{\lambda} / R \underline{m}_{e}^{3}\right]\left[1 / R_{\lambda}^{2} @^{4}\right]=1 / R \underline{m}_{e}^{3} R_{\lambda} @^{4} \\
\underline{a}=1 / R^{1 / 3} \underline{m}_{e} R_{\lambda}^{1 / 3} @^{4 / 3}=3.188747033 E-11 / R_{\lambda}^{1 / 3}
\end{gathered}
$$

or $x K_{M}=4.655043568 E-28[\mathrm{M}] / R_{\lambda}^{1 / 3}$

For $\mathrm{H}_{1}$ ionization energy, find $\underline{R}_{\lambda}$ :

$$
\begin{gathered}
\underline{m}_{e}^{3} / 2=2.381564435 E 19=\underline{h} \omega_{\lambda}=2 \pi \underline{m}_{e}^{3} \underline{c} / \underline{\lambda} \\
\underline{\lambda}=2 \pi \underline{m}_{e} / R_{\lambda} @ \\
\underline{m}_{e}^{3} / 2=2 \pi \underline{m}_{e}^{3} \underline{m}_{e} @ \underline{R}_{\lambda} @ / 2 \pi \underline{m}_{e}=\underline{m}_{e}^{3} \underline{R}^{2} \underline{R}_{\lambda} \\
\underline{R}_{1}=1 / 2 @^{2}=2.662567725 E-5 \\
\underline{m}_{\lambda}=\underline{R}_{\lambda} \underline{m}_{e}=\underline{m}_{e} / 2 @^{2}=96.51573166 \\
\underline{a}_{1}=3.188747034 E-11 / \underline{R}_{1}^{1 / 3}=1.067873588 E-9 \\
\underline{\lambda}_{1}=2 \pi \underline{m}_{e} / R_{1} @=4 \pi \underline{m}_{e} @=4 \pi \underline{c}=6.242261793 E 9 \text { Note this equivalence! } \\
x K_{M}=9.112670372 E-8[\mathrm{M}][911.267037 \text { Ang }] \\
\underline{c}_{\lambda 1}=\underline{m}_{e} @ 2 \pi / \underline{R}_{\lambda 1} @{ }^{2}=4 \pi \underline{m}_{e} @=6.242261792 E 9
\end{gathered}
$$

or $x K_{M}=1.558918919 E-26[\mathrm{M}]$

or $x K_{M}=9.112670372 E-8[\mathrm{M}]$ [911.267037 Ang]

\section{Cosmic Background Radiation as gravitational binary}

$$
\begin{gathered}
R_{S}=1089 \quad \lambda_{c b}=1.063 E-3 \mathrm{~m} \quad 1.063 E-3 \mathrm{~m} / 1089=0.9761 E-6 \mathrm{~m} \\
\underline{\lambda}_{c b}=1.063 E-3 \mathrm{~m} / K_{M}=7.28164634 E 13=5.541593868 \underline{m}_{e}^{2} \\
\underline{\lambda}_{c b}=\underline{c}_{d B}=\underline{m}_{e} @ 2 \pi / R_{c b} @{ }^{2}=2 \pi \underline{m}_{e} / R_{c b} @=5.541593868 \underline{m}_{e}^{2} \\
\underline{R}_{c b}=2 \pi \underline{m}_{e} / @ 5.541593868 \underline{m}_{e}^{2}=1 / @ 0.881972057 \underline{m}_{e} \\
=1.133822769 / @ \underline{m}_{e}=2.282511948 E-9 \\
\underline{R}_{1} / \underline{R}_{c b}=1.166507683 E 4 \\
\underline{m}_{\lambda}=\underline{R}_{c b} \underline{m}_{e}=1.133822769 / @ \text { near electron neutrino mass } \\
\underline{a}_{c b}=1 / R^{1 / 3} \underline{m}_{e} R_{c b}^{1 / 3} @{ }^{4 / 3}=3.188747034 E-11 / R_{c b}^{1 / 3} \quad R_{c b}^{1 / 3}=1.316652059 E-3 \\
\underline{a}_{c b}=2.421860065 E-8 \text { or } \times K_{M}=3.535515362 E-25 \mathrm{~m}
\end{gathered}
$$

\section{Conclusions}

The gravitational binary orbit radius solutions for photons from the Hydrogen ground state and the cosmic background are physically possible. All fundamental particles can be equal or unequal binaries. Binary structure is characteristic of all life. 


\section{Complexity in Physics}

In the last fifty years an enormous amount of information has been acquired about Nature and the Universe. Because of the static point of view of the Standard Model of Physics, much of the new information would be classified as "emergent phenomena".

The dominant view of complexity is that evolving forms are disconnected from the causality that characterizes traditional Physics. This static viewpoint bypasses an obvious alternative: creativity is inherent in the fundamental structure of causality; emergent phenomena are an inherent part of the evolutionary process of physics.

The limiting of communication velocity to the velocity of light is obviously contradicted by non-locality in quantum mechanics. US9 provides an excellent opportunity to consider an addition to the Standard Model which provides flexibility.

Fundamental particles are artifacts of evolution. Each represents a quantum state of the evolution of the Universe. Perhaps all quantum states are artifacts of universal evolution, representing epochs in the development of the Universe. Therefore, physics must develop an evolutionary sequence of logical events that involve all of the fundamental parameters of Physics.

Fundamental particles live in the same Universe as humans, and share similar properties and evolutionary requirements. Survival for fundamental particles requires maintaining a defined level of communication. To exist is to communicate. The communication scheme is also subject to evolution and probably consists of several levels. E\&M and gravity are separate levels. Angular momentum and nuclear forces are probably two more levels. These levels are separated by digital frequencies and communication velocities which do not normally interact in the internal processing.

Digital calculations internal to particles result in the reaction of the particles to the fields of Standard Physics. This requires a balance between external fields and internally generated actions. This is a concept inherent in conservation of momentum and energy.

In US9, the concept of zero is equivalent to non-existence and therefore is excluded from the physics of nature. Standard Physics has required the existence of zero because of the arbitrary units. US9 assumes the existence of a minimum digit (1), the smallest entity in Nature, yet to be determined. As a consequence, the concept of mathematical infinity is non-existent. However, all other real numbers are valid, but they are expected to have defined physical minimum and maximum values.

\section{The Organizational Principle}

The Uncertainty Principle is normally assumed to be totally statistically random. This is impossible to verify and is probably not true. A better statement is "The Uncertainty Principle is also the Organizational Principle". It is obvious that this 
hypothesis requires evaluating what fraction of $+/-\hbar$ is available for nonrandom action under what interaction conditions. In US9-1, a unitless transformation of $\hbar$ puts momentum and distance on the same scale which enables a discussion of a causal component in the U.P.

The causal component of the U.P. occurs when [+ or - or 0$]$ is a decision reaction with interacting particles which results in a momentum change parallel or anti-parallel to the momentum of the interacting particle. The result appears random when the incoming particle momenta are random. The accumulated effects of many such decisions can accumulate to produce stream motion $[+]$ or orbital motion [-]. This is the primary cause of the dominance of order in atoms, star systems and galaxies. This also results in a common explanation for jet streams, tornadoes, eddies, hurricanes, spiral galaxies, galactic jets and magmatic plumes. Standard Physics provides an excellent description of all such events, but it does not provide a causal description, a physical step-by-step evolution that produces these phenomena.

The $[+]$ events, resulting in currents (streaming) are fairly intuitive. In the Hydrogen atom, we can easily see the result of the [-] events in the Bohr description where the electron [-] events have interacted with the proton's motion, thus producing conservation of angular momentum.

The recent discovery of giant magmatic plumes cooling the core of the earth is not predicted by Standard Physics, but can be with the OP.

The Organizational Principle enables production and maintenance of atomic and molecular structures via a bias towards larger organizations.

In order for the OP to operate on cosmic scales in a similar manner, e.g. hurricanes and spiral galaxies, tornadoes and galactic jets, the communication time between particles must be shorter than that provided by the velocity of light. This is also clearly required to explain the communication required to produce Newton's Laws and Mach's Principle (Einstein's Failure) and quantum mechanical non-locality.

Angular momentum conservation is the dominant expression of order in the Universe. The similar morphology of tornadoes and galactic jets, hurricanes and spiral galaxies, atoms and binary stars, etc. suggest a physical law which spans the enormous size scale. This requires a communication time much shorter than that available with communication via the velocity of light.

The magnetic field is the equivalent of the Organizing Principle as the individual particles move in response to the dominant motion of other charged particles.

\section{Summary}

The possibility has been established that fundamental particles can be composed of gravitational binaries. The proton can have a stable structure which contains the fundamental laws of Physics in the form of internal processing. Also, if the Uncertainty Principle can be viewed as the Organizational Principle via enabling 
the assumed randomness to contain significant ordered action which creates evolution to greater complexity, then the laws of Physics become the basis of biological evolution. The OP is an internal particle processing system.

The above work contains a new way of looking at Physics and many clues to a more complex system of fundamental particle communication which enables evolution of Physics as a base for biological evolution.

Evolving Physics creates complexity. Nature is never as simple as we imagine it to be. Although we discover that Laws of Physics are amazingly simple, they will always be found to have limits where complexity increases. Physicists must always be searching for alternative viewpoints, otherwise the search for truth becomes stagnant.

Experience of Nature is Universal, we have inherited this from the Hydrogen atom. We are a part of fundamental Physics.

\section{References}

[1] Christy, J.W. (2015) Unitless Physics I: Relating the Fundamental Constants. International Journal of Astronomy and Astrophysics, 5, 182-192. https://doi.org/10.4236/ijaa.2015.53023

[2] Smolin, L. (2006) The Trouble with Physics. Houghton Mifflin Company, Boston, 349.

[3] Lincoln, D. (2012) Understanding the Universe. World Scientific, Singapore, 190. https://doi.org/10.1142/8313

[4] Bowman, G.E. (2011) Essential Quantum Mechanics. Oxford University Press, Oxford, 91.

[5] Yang, F. and Hamilton, J.H. (2010) Modern Atomic and Nuclear Physics. World Scientific, Singapore, 664.

[6] Schlegel, R. (1980) Superposition and Interaction. University of Chicago Press, Chicago, 171.

[7] Van Flandern, T. (1993) Dark Matter, Missing Planets and New Comets. North Atlantic Books, Berkeley, 51.

[8] Henley, E.M. and Garcia, A. (2007) Subatomic Physics. World Scientific, Singapore, 115. https://doi.org/10.1142/6263

[9] MacGregor, M.H. (2007) The Power of Alpha. World Scientific Publishing, Singapore, 338 . 
Submit or recommend next manuscript to SCIRP and we will provide best service for you:

Accepting pre-submission inquiries through Email, Facebook, LinkedIn, Twitter, etc. A wide selection of journals (inclusive of 9 subjects, more than 200 journals)

Providing 24-hour high-quality service

User-friendly online submission system

Fair and swift peer-review system

Efficient typesetting and proofreading procedure

Display of the result of downloads and visits, as well as the number of cited articles Maximum dissemination of your research work

Submit your manuscript at: http://papersubmission.scirp.org/

Or contact jmp@scirp.org 